$\begin{array}{ll}\text { Research Square } & \begin{array}{l}\text { Preprints are preliminary reports that have not undergone peer review. } \\ \text { They should not be considered conclusive, used to inform clinical practice, } \\ \text { or referenced by the media as validated information. }\end{array}\end{array}$

\title{
Ethnobotanical Survey of Local Wisdom Knowledge on Medicinal Plants Used by the Traditional Phouthai Ethnic Group in NaKeu Village, Hinboun District, Khammouan Province, Lao PDR
}

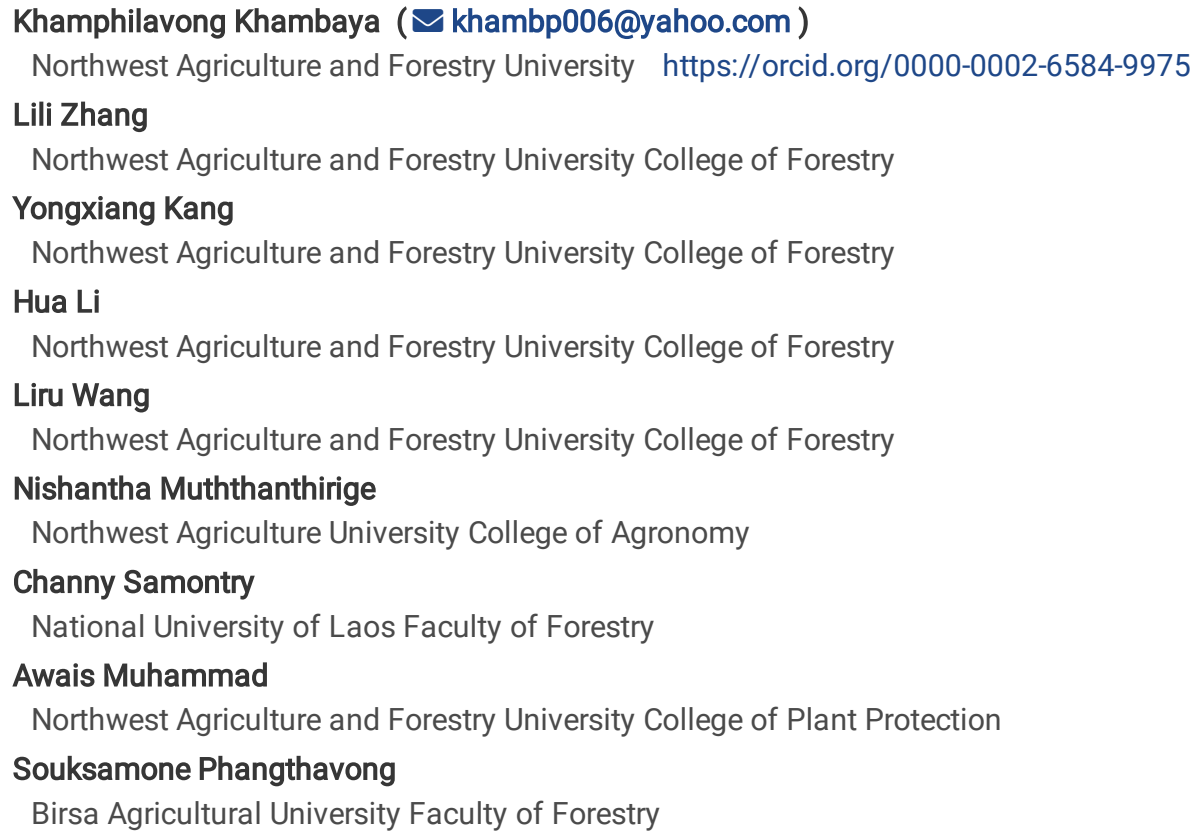

Research

Keywords: Ethnobotaniacl, Medicinal Plants, Traditional local wisdom, Plant parts used and Treatment

Posted Date: September 21st, 2020

DOI: https://doi.org/10.21203/rs.3.rs-54642/v1

License: (c) (i) This work is licensed under a Creative Commons Attribution 4.0 International License. Read Full License 


\section{Abstract}

Background: Plants have been a part of rural area's people life since prehistoric time, plants is important in the traditional cultures in the worldwide where human used it from birth to end of life. Idigen

This study was carried out to explore and recorded the plants and part used of plants for treating numerous people illness and disease by the traditional healers of the Phouthai ethnic group in Nakeu village, Hinboun district, Khammouan province, middle part Lao PDR for medicinal plants purposes. The current study was conducted, first, to identify plant species used as a remedy for human illness, diseases and health improve, and secondly to determine plant parts used, the technique of preparation and religious belief on the preferred source of healthcare an around Hinboun district, Khammouane province, Lao PDR.

Methods: Ethnobotanical data for this paper were gathering from 03 traditional healers, and 69 households, therefore including the household of both gender (husband or wife), and across different level of education, age group, as well as the level of experience and knowledge of medicinal plants use. Plant specimens were collected during field survey which had been collected from natural wild plant and home gardens, information on each medicinal plant was recorded by using the data capture form.

Results: We found 79 medicinal plants species, 51 families, and can't identifies 2 species. Among the tree 44 species, $55.70 \%$; shrub 20 species, $25.32 \%$; liana 12 species, $15.19 \%$; and gasses 3 species, $3.8 \%$, were used to treat 34 sicknesses. A designation of all recorded species including the plant family names, dialect names, part used, and process to preparation of treatment. The roots are the plant part most commonly used (36.70\%), followed by the stem (25.31\%), leaves (10.12\%), liana (10.12\%), whole plant (6.32\%), fruits (6.32\%), and bark (3.79\%). The medicinal plants are prepared using dried plant parts while some other species are using in fresh form. Boiling, soaking with cold water or alcohol, crushing, and burning are main methods of preparation.

Conclusion: In this study, 79 medicinal plant species were documented as use in the treatment of different common sickness including stomach problem (gastrointestinal, flatulence, diarrhea, detox), kidney infections, fever, dysmenorrheal postpartum tonic, nervous system, skin disease, health tonic, lever infections, heart disease, dressing wounds, and others.

\section{Introduction}

Plants have been a part of rural area's people life since prehistoric time, plants is important in the traditional cultures in the worldwide where human used it from birth to end of life (De Boer and Lamxay,2009; Lamxayet al, 2011). Medicine is the very important plant resource for people and constructions are built from different plant species (Pieroniet al., 2017). Plant derived medicine is used to treating illness and disease, health tonic and are important in primary healthcare around the world (Elkinget al., 2009), especially the people who are live in rural area is far from hospital. Even though, High percentage of the population also in developed countries such as Austraria, Canada, France, Belgium, America use traditional medicine (McFarland et al., 2002; Molassiotiset al., 2005). As present time used modern medicines were formerly resolved or synthesized on the basis of plant derivatives which serves as a stratagem (Fabricant and Farnsworth, 2001; Iwu, 2002; Lu et al., 2011),looking for bioactive compounds from plants consumes large amounts of time, specialists, and funding, and the look for traditional medicinal plants can, therefore, help to reduce costs for pharmacy discoveries. At the same time, traditional knowledge is being eroded by several processes related to globalization and urbanization(Ragupathyet al., 2008; Srithiet al., 2009).As people in each region of the world have different historical backgrounds, cultures, lifestyles, and since the floras of their environment are different, patterns of their used of plants for medicine also vary, for instance by using different plant species and plant parts, methods of preparations, and applications(Kichuet al., 2015;Menaleet al., 2016).

The use of a plant for medicine is normally strictly related to a specific part of the plant such as roots, stems, leaves (Asowatata-Ayodeteet al., 2016, Ouelbaniet al., 2016, Agbodekeet al., 2016, Birruet al., 206), although some mode preparations can use any or all parts of a plant. The culling of plant part will often depend on which organ has the highest concentration of the bioactive substance that functions in the treatment (Abbasiet al., 2010, Abbas et al., 2016, Chekole., 2017, Dhamaet al., 2018), but since not all traditional medicine depends on physiological effects other parts may also be used. The preparation of the medicine modifies from lineage, which may use various solvents such as Boiling, soak with water or alcohol (Rametet al., 2018, Asawata-Ayodeleet al., 2016, Lamxayet al., 2011), to crushing without extraction, to ingestions without any preparation. And the medicine may be prepared as a drink or a pill or used without any preparation. Finally, the way of administering the medicine varies from ingestion to inhalation of smoke, to external application to affected parts of the body (Jai-aueet al., 2014, Baruahet al., 2016, Jahandidehet al., 2016). All these variations have cultural, geographic and temporal dimensions.

The rural people almost exclusive on traditional medicine for their primary healthcare, their dependence on medicine is high due to lack of modern healthcare services (Lu et al, 2011, Delang. 2007). Even though the communities have known and used a lot of medicine plants, but no one has written their knowledge for future use (Pooma and Suddee, 2014, Hidayatiet al., 2015). The most of local knowledge which are still available among traditional healers is another be lost or passed to next young generation only by the oral word (Park et al., 2018). Besides, lack of adequate referent on knowledge of ethnomedicinal plants use among often cited problems in the study site (Birhanuet al., 2015, Staubet al., 
2015). The current study was conducted, first, to identify plant species used as a remedy for human illness, diseases and health improve, and secondly to determine plant parts used, the technique of preparation and religious belief on the preferred source of healthcare an around Hinboun district, Khammouane province, Lao PDR.

\section{Materials And Methods}

\subsection{Study area}

The Khammouane province, one of a province in middle part of Lao PDR as presented in Fig. 1. The land area of $16,315 \mathrm{~km}^{2}$, and is almost of forest mountain terrain. The province share bordered with Savannakhet province to the south, Bolikhamxay province to the north and northwest, Thailand to the west, and Vietnam to the east. The forest area encloses of three store areas. These are the PhouHinPhoun National Biodiversity Conservation Area with an area is 150,000 ha, Nakai-Nam Thern National Biodiversity Conservation Area has cover areas is 352,200 ha, and Hin Nam No National Biodiversity Conservation Area with an areas 86,229 ha.

The study was conducted in Nakeu village, Hinboundistrict. In this part of Khammouane province traditionally inhabited by the Phouthai people. It is located $33 \mathrm{~km}$ far away from the town, the geographically situated between $17^{\circ} 64^{\prime}-86^{\circ} 78^{\prime} \mathrm{N}$ and $140^{\circ} 78^{\prime}-48^{\circ} 57^{\prime} \mathrm{E}$, the total land area of 35,863 ha (Fig. 1). The mean monthly temperature is $32{ }^{\circ} \mathrm{C}$, ranging from $15^{\circ} \mathrm{C}$ to $38^{\circ} \mathrm{C}$,and total rainfall is $1200 \mathrm{~mm}$, ranging from 600 to $1600 \mathrm{~mm}$ (Statistical Authority, 2017). The population of the study site is amounted to 960 people, female is 465 people all most of them is Phouthai people.

\subsection{Ethnobotanical data collection}

Looking at the traditional healer in the Hinboun district area was conducted with the first agreement of citation by community leaders of each household. Our research team started an interaction with each expected respondent by explaining the aim of the study in order to solicit their consent and co-operation before any ethnobotanical data gathering. While this group discussion the research team was emphasized the extensive value which each traditional healer's contribution can make the compilation a recorded of traditional knowledge of medicinal plants in Hinboun district area.

Whit the helping of a commenter, all interviews and discussions were conduction in Nakeu village. Ethnobotanical data for this paper were gathering from 03 traditional healers, and 69 households, therefore including the household of both gender (husband or wife), and across different level of education, age group, as well as the level of experience and knowledge of medicinal plants use. In every part of the field survey, semi-structured interviews and group discussions were conducted in order to collect the local wisdom information about medicinal plants. The question was designed to focus on the local names of plants, their multitudinous medicine application, the part of used, the methods of preparation, and handle treatment to patients.

\subsection{Plant collection and plant identification}

The field survey was undertaken with randomly selection household on a descriptive statistics was employed to analyze and summarize the data on report medicinal plants, part of used, mode of preparation, ministry, the postulate of used, habitats of medicinal plant, and influence of realization, religious beliefs and culture on the extra source of healthcare. Plant specimens were collected during field survey which had been collected from natural wild plant and home gardens, information on each medicinal plant was recorded by using the data capture form. Certain medicine plants were identified in the field and remaining ones identified through published floras and another reference. After identifying medicinal plants, a photograph and herbarium specimen of each medicinal plant was taken in the herbarium at Faculty of Forestry Science, the National University of Laos for comparison and verification of each plant's scientific name.

\section{Result And Discussion}

\subsection{Medicinal plants species}

From this research it was found that among the 33 respondents who were interviewed, the majority was falling within the villagers ( 30 people) and healers ( 3 people). The total of 79 species, and 51 families, and can't identifies 2 species. Among the tree 44 species, 55.70 percentage; shrub 20 species, 25.32 percentage; liana 12 species, 15.19 percentage; and grasses 3 species, 3.8 percentage (Table 1) wereusedto treat 34 sicknesses. A designation of all recorded species including the plant family names, dialect names, part used, and process to preparation of treatment show in Table 1.

The study indicated 32 species (40.50\%) that are used for medicinal purposes by traditional healers, 13 species (16.45\%) are used by villagers, and 34 species (43.03\%) used by both (healers and villagers) (Fig. 2). 
Plants are most frequently used for the treatment of the sickness such as stomach problems, nervous system, kidney infection, liver tonic, menstrual cycle, and postpartum tonic (55.69\%) which shown in Fig. 3 and Table 1. These results are followed by the pattern found in medicinal plant studies around the world, such as in Brazil (Bolson et al., 2015), India (Kichuet al., 2015), Italy (Fortini., 2016). The roots are the plant part most commonly used in this community (36.70\%), followed by the stem (25.31\%), leaves (10.12\%). leaves are commonly used like medicine in many parts in developing countries (Yemeleet al., 2015; Mukunguet al.,2016; Fortiniet al., 2016), and using leaves for medicine is a sustainable way of using plants (Mukunguet al., 2016). From this research it is concluded that the liana (10.12\%),whole plant (6.32\%), fruits (6.32\%), and bark (3.79\%) were used for treatments which shown in Fig. 4, the most used part of the plant are roots(35\%), because of their availability throughout the year, traditionally considered to be strong medicine, have a good smell and easy to drink. the roots are being used in different medicine manufacturing more frequently, because sometime it's difficult to use other plant parts due to presence of phytochemecal compounds (Singh et al., 2016)

The medicinal plants listed in Table 1 are used for the treatment of 34 sicknesses. Most of the villagers and traditional healers make medicine by boiling and distilling medicinal plants, that methods were also used by (Gadisaet al., 2018, Metheeet al., 2018, Mahwasaneet al., 2013). Medicine preparation methods of dominate treatments vary from drying and burning the plants for treating warts with the smoke and crushing. (Alemayehuet al., 2015, Chekoleet al., 2017, Girmay and Teshome 2017) in their study also mention the mode of remedy preparation to the charred remains are used as a dressing on wounds.

The villagers and traditional healers usually collected the medicinal plants from different fields, plans parts were dry and crushed before storing in bag or bottles. This method is also similar with (Gadisaet al., 2018). It is very difficult for patients to recognize plants species that are used for their treatment, which sometimes seem ordinary plants that growing in gardens or in field. Some villagers prefer to store plants material in dry or powdered form inside bottles or plastic bags to reduce the field collection trips and make sure the availability of plants part possible throughout the year. 
Table 1

Ethnobotanical information of medicinal plants uses by villagers and healers to treat sickness in Nakeu village, Hinboundistric, Khammouane province, Laos.

\begin{tabular}{|c|c|c|c|c|c|c|c|c|}
\hline No & Dialect names & Scientific name & Family name & References & $\mathrm{vg} /\left.\mathrm{h}\right|^{\star \star}$ & $\begin{array}{l}\text { Plant } \\
\text { form }\end{array}$ & $\begin{array}{l}\text { Part } \\
\text { used }\end{array}$ & $\begin{array}{l}\text { Method of } \\
\text { prepare }\end{array}$ \\
\hline 1 & NaoNarm & $\begin{array}{l}\text { Artabotrys spinosus } \\
\text { Craib }\end{array}$ & Annonaceae & $\begin{array}{l}\text { Onvilay et } \\
\text { al. } 2016\end{array}$ & hl & tree & $\mathrm{t}$ & $\begin{array}{l}\text { The stem is } \\
\text { boiled and } \\
\text { mixture drank } \\
\text { to treating } \\
\text { kidney } \\
\text { infection. }\end{array}$ \\
\hline 2 & Theangseang & Cananga latifolia & & $\begin{array}{l}\text { Southavong } \\
\text { et al. } 2014\end{array}$ & both & tree & $r$ & $\begin{array}{l}\text { Roots are } \\
\text { soaked in cold } \\
\text { water for } 2- \\
3 \text { day drank to } \\
\text { bring down } \\
\text { fever }\end{array}$ \\
\hline 3 & Som Lom & $\begin{array}{l}\text { Aganonerion } \\
\text { polymorphum P. }\end{array}$ & Apocynaceae & $\begin{array}{l}\text { Onvilay et } \\
\text { al. } 2016\end{array}$ & hl & liana & $r$ & $\begin{array}{l}\text { Roots are boiled } \\
\text { and mixture } \\
\text { drank to } \\
\text { treating kidney } \\
\text { stone. }\end{array}$ \\
\hline 4 & Chum Pha & Plumeri alba & & $\begin{array}{l}\text { Onvilay et } \\
\text { al. } 2016\end{array}$ & $\mathrm{vl}$ & tree & $r$ & $\begin{array}{l}\text { The roots are } \\
\text { boiled and } \\
\text { drank for } \\
\text { nervous pain. }\end{array}$ \\
\hline 5 & Teen Phet & Alstonia scholaris L. & & $\begin{array}{l}\text { Newman et } \\
\text { al. } 2007\end{array}$ & $\mathrm{vl}$ & tree & $\mathrm{t}$ & $\begin{array}{l}\text { The stem is } \\
\text { boiled and } \\
\text { mixture drank } \\
\text { to treating } \\
\text { paralysis. }\end{array}$ \\
\hline 6 & Puk Nork & $\begin{array}{l}\text { Centella asiatica }(\mathrm{L}) \\
\text { Urbon }\end{array}$ & Apiaceae & $\begin{array}{l}\text { Lamxay et } \\
\text { al. } 2011\end{array}$ & $\mathrm{vl}$ & shrub & wh & $\begin{array}{l}\text { The whole plant } \\
\text { boiled and } \\
\text { mixture drank } \\
\text { for alleviating } \\
\text { numbness, } \\
\text { aches and } \\
\text { pains in the } \\
\text { body. }\end{array}$ \\
\hline 7 & Pha Rai & $\begin{array}{l}\text { Schefflera elliptica } \\
\text { (Blume) Harms }\end{array}$ & Araliaceae & $\begin{array}{l}\text { Southavong } \\
\text { et al. } 2014\end{array}$ & $\mathrm{hl}$ & tree & $r$ & $\begin{array}{l}\text { The roots are } \\
\text { boiled and } \\
\text { drank for } \\
\text { stomach } \\
\text { problems. }\end{array}$ \\
\hline 8 & Kok Tang Kai & $\begin{array}{l}\text { Scheffera octophylla } \\
\text { (Loureiro) Harms }\end{array}$ & & $\begin{array}{l}\text { Southavong } \\
\text { et al. 2013, } \\
2014\end{array}$ & both & tree & $\mathrm{t}$ & $\begin{array}{l}\text { Stem cut is } \\
\text { small piece are } \\
\text { soaked with } \\
\text { alcohol and } \\
\text { drank for } \\
\text { nervous } \\
\text { system. }\end{array}$ \\
\hline 9 & Ya Far Rang & $\begin{array}{l}\text { Chromolaena odorata } \\
\text { (L.) R. King \& H. } \\
\text { Robinson }\end{array}$ & Asteraceae & $\begin{array}{l}\text { Lamxay et } \\
\text { al. } 2011\end{array}$ & $\mathrm{vl}$ & shrub & $\mathrm{t}$ & $\begin{array}{l}\text { The stem is } \\
\text { boiled and } \\
\text { mixture drank } \\
\text { for stomach } \\
\text { detox }\end{array}$ \\
\hline 10 & Warn Hang & Aloe vera $\mathrm{L}$. & Asphodelaceae & $\begin{array}{l}\text { Southavong } \\
\text { et al. } 2013\end{array}$ & both & tree & r & $\begin{array}{l}\text { Roots are } \\
\text { soaked with } \\
\text { alcohol and } \\
\text { mixture drank } \\
\text { for sexual } \\
\text { performance. }\end{array}$ \\
\hline 11 & Mai Khea Laow & $\begin{array}{l}\text { Haplophrama } \\
\text { adenophyllum }\end{array}$ & Bignonaceae & $\begin{array}{l}\text { Southavong } \\
\text { et al. } 2013\end{array}$ & both & tree & $r$ & $\begin{array}{l}\text { Roots are boiled } \\
\text { and mixture } \\
\text { drank for } \\
\text { stomach } \\
\text { problems. }\end{array}$ \\
\hline
\end{tabular}

Remake: vg* villagers, hl* healers; t, stem; l, leaves; r, roots; wh, whole plant; f, fruit; b, bark; In, liana. 


\begin{tabular}{|c|c|c|c|c|c|c|c|c|}
\hline No & Dialect names & Scientific name & Family name & References & $\mathrm{vg} / \mathrm{h} \mathbf{h}^{* *}$ & $\begin{array}{l}\text { Plant } \\
\text { form }\end{array}$ & $\begin{array}{l}\text { Part } \\
\text { used }\end{array}$ & $\begin{array}{l}\text { Method of } \\
\text { prepare }\end{array}$ \\
\hline 12 & Lin Mai & $\begin{array}{l}\text { Oroxylum indicum (L.) } \\
\text { Kurz }\end{array}$ & & $\begin{array}{l}\text { Newman et } \\
\text { al. } 2007\end{array}$ & $\mathrm{vl}$ & tree & $\mathrm{t}$ & $\begin{array}{l}\text { The stem is } \\
\text { boiled and } \\
\text { mixture drank } \\
\text { to treating } \\
\text { emaciation. }\end{array}$ \\
\hline 13 & Nard Narm & Buddleja asiatica Lour. & Buddlejaceae & $\begin{array}{l}\text { Southavong } \\
\text { et al. } 2013\end{array}$ & both & shrub & $r, t$ & $\begin{array}{l}\text { Roots are boiled } \\
\text { and mixture } \\
\text { drank for } \\
\text { aweary, post- } \\
\text { partum tonic, } \\
\text { cough and } \\
\text { bruises. }\end{array}$ \\
\hline 14 & Sakharm & Peltophorum dasyrachis & Casalpiniaceae & $\begin{array}{l}\text { Southavong } \\
\text { et al. } 2013\end{array}$ & both & tree & $r$ & $\begin{array}{l}\text { Roots are } \\
\text { boiled/soaked } \\
\text { with alcohol } \\
\text { drank for } \\
\text { infertility, } \\
\text { dysmenorrhoea } \\
\text { and to } \\
\text { improved } \\
\text { sexual } \\
\text { performance }\end{array}$ \\
\hline 15 & Mai Tew & $\begin{array}{l}\text { Cratoxylonformorsum } \\
\text { (jacq) Dyer }\end{array}$ & Clusiaceae & $\begin{array}{l}\text { Southavong } \\
\text { et al. } 2013\end{array}$ & $\mathrm{hl}$ & tree & $\mathrm{t}$ & $\begin{array}{l}\text { The stem is } \\
\text { boiled or } \\
\text { soaked with } \\
\text { alcohol and } \\
\text { drank for } \\
\text { nourish the } \\
\text { body. }\end{array}$ \\
\hline 16 & Mark KharmKheu & Cnestisrmiflora Griff & Connaraceae & $\begin{array}{l}\text { Southavong } \\
\text { et al. } 2013\end{array}$ & both & liana & In & $\begin{array}{l}\text { Liana are boiled } \\
\text { or soaked with } \\
\text { alcohol and } \\
\text { drank for health } \\
\text { tonic }\end{array}$ \\
\hline 17 & HuoaEung & Costusspeiosus(Koecnig) & Costus & $\begin{array}{l}\text { Onvilay et } \\
\text { al. } 2016\end{array}$ & hl & shrub & $\mathrm{t}$ & $\begin{array}{l}\text { The stem is } \\
\text { boiled and } \\
\text { mixture drank } \\
\text { to treating } \\
\text { kidney } \\
\text { infection. }\end{array}$ \\
\hline 18 & Mark Keae & $\begin{array}{l}\text { Combretum } \\
\text { quagrangulare Kurz }\end{array}$ & Combretaceae & $\begin{array}{l}\text { Southavong } \\
\text { et al. } 2014\end{array}$ & both & tree & $t, l$ & $\begin{array}{l}\text { Stem and } \\
\text { leaves crushed } \\
\text { rubbed on body } \\
\text { or used fruits } \\
\text { and leaves are } \\
\text { boiled as bath } \\
\text { to treating rash } \\
\text { on body/anti- } \\
\text { allergy. }\end{array}$ \\
\hline 19 & Han Drang & Artocarpu ssp. & & $\begin{array}{l}\text { Newman et } \\
\text { al. } 2007\end{array}$ & hl & tree & $f$ & $\begin{array}{l}\text { The dry's fruits } \\
\text { are boiled and } \\
\text { mixture drank } \\
\text { to alleviating } \\
\text { jaundice }\end{array}$ \\
\hline 20 & KraDeang & $\begin{array}{l}\text { calycopteris floribunda } \\
\text { (Roxn.) lamk. }\end{array}$ & & $\begin{array}{l}\text { Southavong } \\
\text { et al. } 2014\end{array}$ & $\mathrm{hl}$ & liana & $\ln$ & $\begin{array}{l}\text { The liana is } \\
\text { boiled and } \\
\text { drank for bring } \\
\text { down fever. }\end{array}$ \\
\hline 21 & Ya Khum Pao & Carexbaccans Nees. & Cyperaceae & $\begin{array}{l}\text { Onvilay et } \\
\text { al. } 2016\end{array}$ & hl & gasses & $r$ & $\begin{array}{l}\text { Roots are boiled } \\
\text { and mixture } \\
\text { drank to } \\
\text { treating kidney } \\
\text { problems. }\end{array}$ \\
\hline
\end{tabular}

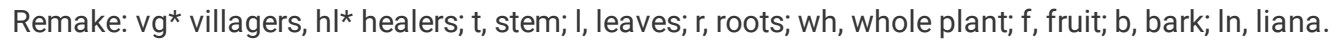




\begin{tabular}{|c|c|c|c|c|c|c|c|c|}
\hline No & Dialect names & Scientific name & Family name & References & $\mathrm{vg} * / \mathrm{h} \mid * *$ & $\begin{array}{l}\text { Plant } \\
\text { form }\end{array}$ & $\begin{array}{l}\text { Part } \\
\text { used }\end{array}$ & $\begin{array}{l}\text { Method of } \\
\text { prepare }\end{array}$ \\
\hline 22 & Hark Kha Jai & Cyperuselatus L. & & $\begin{array}{l}\text { Southavong } \\
\text { et al. 2013, } \\
2014\end{array}$ & both & shrub & $\mathrm{t}$ & $\begin{array}{l}\text { Stem are boiled } \\
\text { and mixture } \\
\text { drank to stop } \\
\text { diarrhea. }\end{array}$ \\
\hline 23 & Khean Hin & Hopea ferreapierre & Dipterocarpaceae & $\begin{array}{l}\text { Hua \&Tzen- } \\
\text { Yuh } 2017\end{array}$ & $\mathrm{hl}$ & tree & b & $\begin{array}{l}\text { Bark are soaked } \\
\text { with cold water } \\
\text { or boiled and } \\
\text { drank for } \\
\text { alleviating } \\
\text { diarrhea. }\end{array}$ \\
\hline 24 & Thonpheang & Dillenia baillonii & Dilleniaceae & $\begin{array}{l}\text { Southavong } \\
\text { et al. } 2014\end{array}$ & both & tree & $r$ & $\begin{array}{l}\text { Roots are } \\
\text { soaked in cold } \\
\text { water for 2- } \\
3 \text { day drank to } \\
\text { bring down } \\
\text { fever }\end{array}$ \\
\hline 25 & Mai Mark San & Dillenia Sp. & & $\begin{array}{l}\text { Southavong } \\
\text { et al. } 2014\end{array}$ & $\mathrm{hl}$ & tree & $r$ & $\begin{array}{l}\text { Roots are boiled } \\
\text { and mixture } \\
\text { drank to } \\
\text { treating liver } \\
\text { disease. }\end{array}$ \\
\hline 26 & KeauKra & Diospyros mollis & Ebenaceae & $\begin{array}{l}\text { Southavong } \\
\text { et al. } 2013\end{array}$ & $\mathrm{hl}$ & tree & $r$ & $\begin{array}{l}\text { The roots boiled } \\
\text { or soaked with } \\
\text { alcohol and } \\
\text { drank for } \\
\text { nourish the } \\
\text { body or health } \\
\text { tonic. }\end{array}$ \\
\hline 27 & Mai Moun & Elaeocarpus siamensis & Elaeocarpaceae & $\begin{array}{l}\text { Hua \&Tzen- } \\
\text { Yuh } 2017\end{array}$ & both & tree & $r$ & $\begin{array}{l}\text { Roots are boiled } \\
\text { and mixture } \\
\text { drank for } \\
\text { regulating } \\
\text { menstrual } \\
\text { cycle. }\end{array}$ \\
\hline 28 & Hoo Shang & $\begin{array}{l}\text { Macaranga denticulate } \\
\text { (Blume) }\end{array}$ & Euphorbiaceae & $\begin{array}{l}\text { Lamxay et } \\
\text { al. } 2011\end{array}$ & both & shrub & I & $\begin{array}{l}\text { The leaves are } \\
\text { boiled and } \\
\text { drank for } \\
\text { stomach } \\
\text { problem. }\end{array}$ \\
\hline 29 & Mark Yao & Vernicia montana LOUR. & & $\begin{array}{l}\text { Southavong } \\
\text { et al. } 2013\end{array}$ & $\mathrm{vl}$ & tree & $t, 1$ & $\begin{array}{l}\text { Deduct stem or } \\
\text { leaves and used } \\
\text { resin rubbed on } \\
\text { the papilla }\end{array}$ \\
\hline 30 & Kang Pha & $\begin{array}{l}\text { Phyllanthus reticulates } \\
\text { Poir. }\end{array}$ & Euphobiaceae & $\begin{array}{l}\text { Southavong } \\
\text { et al. } 2013\end{array}$ & $\mathrm{hl}$ & tree & I & $\begin{array}{l}\text { The leaves are } \\
\text { soaked with } \\
\text { cold water and } \\
\text { drank for bring } \\
\text { down fever. }\end{array}$ \\
\hline 31 & Mai deang & Xyliakerrii & Fabaceae & $\begin{array}{l}\text { Southavong } \\
\text { et al. } 2013\end{array}$ & both & tree & $r$ & $\begin{array}{l}\text { Roots are } \\
\text { soaked with } \\
\text { cold water and } \\
\text { the mixture } \\
\text { drank for the } \\
\text { postpartum } \\
\text { tonic. }\end{array}$ \\
\hline 32 & None Nai & - & & $\begin{array}{l}\text { Southavong } \\
\text { et al. } 2013\end{array}$ & hl & shrub & $r$ & $\begin{array}{l}\text { Roots are boiled } \\
\text { and mixture } \\
\text { drank to } \\
\text { treating liver } \\
\text { disease, and } \\
\text { nervous } \\
\text { system. }\end{array}$ \\
\hline
\end{tabular}

Remake: vg* villagers, hl* healers; $t$, stem; l, leaves; $r$, roots; wh, whole plant; f, fruit; b, bark; In, liana. 


\begin{tabular}{|c|c|c|c|c|c|c|c|c|}
\hline No & Dialect names & Scientific name & Family name & References & $\mathrm{vg} / \mathrm{hl}{ }^{* *}$ & $\begin{array}{l}\text { Plant } \\
\text { form }\end{array}$ & $\begin{array}{l}\text { Part } \\
\text { used }\end{array}$ & $\begin{array}{l}\text { Method of } \\
\text { prepare }\end{array}$ \\
\hline 33 & Pao Thong & Leptostachya sp. & & $\begin{array}{l}\text { Onvilay et } \\
\text { al. } 2016\end{array}$ & both & tree & $r$ & $\begin{array}{l}\text { Roots are boiled } \\
\text { and mixture } \\
\text { drank to } \\
\text { treating kidney } \\
\text { problems. }\end{array}$ \\
\hline 34 & HomSarmMeuang & Moghanialatifolia & & $\begin{array}{l}\text { Onvilay et } \\
\text { al. } 2016\end{array}$ & hl & liana & $r$ & $\begin{array}{l}\text { The roots are } \\
\text { soaked with } \\
\text { alcohol, and } \\
\text { drank for } \\
\text { improved } \\
\text { sexual } \\
\text { performance, } \\
\text { postpartum } \\
\text { tonic. }\end{array}$ \\
\hline 35 & PukKood Buang & Gleichania linearis & Gleicheniaceae & $\begin{array}{l}\text { Southavong } \\
\text { et al. } 2013\end{array}$ & both & shrub & $r$ & $\begin{array}{l}\text { Roots are boiled } \\
\text { and mixture } \\
\text { drank for } \\
\text { treating } \\
\text { flatulence. }\end{array}$ \\
\hline 36 & KokTha Kai & $\begin{array}{l}\text { Salaciia viminia wallich } \\
\text { ex lawon }\end{array}$ & Hippocrataceae & $\begin{array}{l}\text { Onvilay et } \\
\text { al. } 2016\end{array}$ & hl & shrub & $\mathrm{t}, \mathrm{l}$ & $\begin{array}{l}\text { The stem and } \\
\text { leaves crushed } \\
\text { and rubbed on } \\
\text { body which has } \\
\text { papilla. }\end{array}$ \\
\hline 37 & Mai Ka Sao & Holoptelea intergrifolia & Holoptelea & $\begin{array}{l}\text { Sydara et } \\
\text { al. } 2014\end{array}$ & hl & tree & $r$ & $\begin{array}{l}\text { Roots are boiled } \\
\text { and mixture } \\
\text { drank to } \\
\text { treating liver } \\
\text { disease. }\end{array}$ \\
\hline 38 & Sean Meuang & $\begin{array}{l}\text { Gonocaryum } \\
\text { subrostratum P. }\end{array}$ & Icacinaceae & $\begin{array}{l}\text { Sydara et } \\
\text { al. } 2014\end{array}$ & hl & shrub & $\mathrm{t}$ & $\begin{array}{l}\text { The stem is } \\
\text { soaked with } \\
\text { cold water } \\
\text { drank for } \\
\text { jaundice. }\end{array}$ \\
\hline 39 & PukieTou & Ocimum spp. & Labiaceae & $\begin{array}{l}\text { Southavong } \\
\text { et al. } 2013\end{array}$ & vl & shrub & $\mathrm{t}$ & $\begin{array}{l}\text { The stem is } \\
\text { boiled and } \\
\text { mixture drank } \\
\text { for stomach } \\
\text { detox }\end{array}$ \\
\hline 40 & Ya Nouad Meo & Orthosiphon aristatus & Labiatae & $\begin{array}{l}\text { Onvilay et } \\
\text { al. } 2016\end{array}$ & hl & gasses & wh & $\begin{array}{l}\text { Whole plant is } \\
\text { boiled and } \\
\text { mixture drank } \\
\text { to treating } \\
\text { kidney } \\
\text { problems. }\end{array}$ \\
\hline 41 & Mai Mark Sang & $\begin{array}{l}\text { Litsea cubeba (Lour.) } \\
\text { Pers. }\end{array}$ & Lauraceae & $\begin{array}{l}\text { Southavong } \\
\text { et al. } 2013\end{array}$ & hl & tree & wh & $\begin{array}{l}\text { Whole plants } \\
\text { are boiled and } \\
\text { mixture drank } \\
\text { for purgative, } \\
\text { tonic blood, } \\
\text { malaria, and } \\
\text { dizzy. }\end{array}$ \\
\hline 42 & Zhouangnoy & Cinnamomuminers & & $\begin{array}{l}\text { Lamxay et } \\
\text { al. } 2011\end{array}$ & both & tree & $\mathrm{t}$ & $\begin{array}{l}\text { The stem cut is } \\
\text { a small piece } \\
\text { and boiled } \\
\text { drank for easier } \\
\text { to get } \\
\text { pregnancy. }\end{array}$ \\
\hline 43 & Pha Dong Mod lin & Dalbergia & Leguminoceae & $\begin{array}{l}\text { Southavong } \\
\text { et al. } 2013\end{array}$ & hl & tree & $\mathrm{t}$ & $\begin{array}{l}\text { Stem are boiled } \\
\text { and drank to } \\
\text { treating papilla } \\
\text { on the body. }\end{array}$ \\
\hline
\end{tabular}

Remake: vg* villagers, hl* healers; t, stem; I, leaves; r, roots; wh, whole plant; f, fruit; b, bark; In, liana. 


\begin{tabular}{|c|c|c|c|c|c|c|c|c|}
\hline No & Dialect names & Scientific name & Family name & References & $\mathbf{v g} / \mathrm{h} \mid * *$ & $\begin{array}{l}\text { Plant } \\
\text { form }\end{array}$ & $\begin{array}{l}\text { Part } \\
\text { used }\end{array}$ & $\begin{array}{l}\text { Method of } \\
\text { prepare }\end{array}$ \\
\hline 44 & Sarm Hang & $\begin{array}{l}\text { Moghania macrophylla } \\
\text { (Willdenow) O. Kuntze }\end{array}$ & Leguminosae & $\begin{array}{l}\text { Southavong } \\
\text { et al. } 2013\end{array}$ & $\mathrm{hl}$ & shrub & $\mathrm{t}$ & $\begin{array}{l}\text { Stem are } \\
\text { soaked with } \\
\text { cold water 2- } \\
3 \text { day, drank for } \\
\text { detox stomach. }\end{array}$ \\
\hline 45 & Keua Charn & $\begin{array}{l}\text { Spatholobu sroxburghrr } \\
\text { Benth }\end{array}$ & & $\begin{array}{l}\text { Southavong } \\
\text { et al. } 2013\end{array}$ & $\mathrm{hl}$ & liana & $\ln$ & $\begin{array}{l}\text { The liana is } \\
\text { boiled and } \\
\text { drank for } \\
\text { alleviating sore } \\
\text { throat. }\end{array}$ \\
\hline 46 & Kra Teo & $\begin{array}{l}\text { Pterolobium platypterum } \\
\text { Gagnepain }\end{array}$ & & $\begin{array}{l}\text { Onvilay et } \\
\text { al. } 2016\end{array}$ & $\mathrm{hl}$ & liana & $\ln$ & $\begin{array}{l}\text { The liana is } \\
\text { boiled and } \\
\text { mixture drank } \\
\text { for stomach } \\
\text { problem. }\end{array}$ \\
\hline 47 & Mark Kharm & Tamarindus indicus Linn. & & $\begin{array}{l}\text { Southavong } \\
\text { et al. } 2014\end{array}$ & $\mathrm{vl}$ & tree & $b$ & $\begin{array}{l}\text { The bark is } \\
\text { burned and } \\
\text { made it as the } \\
\text { powder used as } \\
\text { a dressing on } \\
\text { wounds to dry. }\end{array}$ \\
\hline 48 & Khii Leck & Cassia timoriensis Dc. & & $\begin{array}{l}\text { Hua \&Tzen- } \\
\text { Yuh } 2017\end{array}$ & $\mathrm{vl}$ & tree & $t, l$ & $\begin{array}{l}\text { The stem is } \\
\text { boiled and } \\
\text { drank or leaves } \\
\text { cooked dish for } \\
\text { sleep well. }\end{array}$ \\
\hline 49 & Kok Peuay & Lagerstroemia floribunda & Lythraceae & $\begin{array}{l}\text { Newman et } \\
\text { al. } 2007\end{array}$ & hl & tree & $b$ & $\begin{array}{l}\text { Bark soaked } \\
\text { with cold water } \\
15-20 \text { minute } \\
\text { and drank or } \\
\text { leaves are } \\
\text { chewed and } \\
\text { swallowed for } \\
\text { diarrhea. }\end{array}$ \\
\hline 50 & Fai Lang & $\begin{array}{l}\text { Eriolaena candollei } \\
\text { Wallich }\end{array}$ & Malvaceae & $\begin{array}{l}\text { Southavong } \\
\text { et al. } 2013\end{array}$ & $\mathrm{vl}$ & tree & $\mathrm{r}$ & $\begin{array}{l}\text { The roots are } \\
\text { boiled and } \\
\text { drank for } \\
\text { stomach } \\
\text { problems. }\end{array}$ \\
\hline 51 & Keu Haerm & Coscinium fenestratum & Menispermaceae & $\begin{array}{l}\text { Southavong } \\
\text { et al. } 2013\end{array}$ & both & liana & $r$ & $\begin{array}{l}\text { Roots made is } \\
\text { dry and boiled } \\
\text { drank for } \\
\text { gastrointestinal. }\end{array}$ \\
\hline 52 & Keu Khaohor & Tinospora crispa & & $\begin{array}{l}\text { Southavong } \\
\text { et al. } 2013\end{array}$ & both & liana & $\ln$ & $\begin{array}{l}\text { Liana's fresh or } \\
\text { dry are boiled } \\
\text { and drank for } \\
\text { Scabies, } \\
\text { ringworm. }\end{array}$ \\
\hline 53 & $\begin{array}{l}\text { Hum Vang/Hum } \\
\text { Hork }\end{array}$ & Ficushisuta Vahl & Moraceae & $\begin{array}{l}\text { Newman et } \\
\text { al. } 2007\end{array}$ & hl & shrub & r & $\begin{array}{l}\text { Roots are boiled } \\
\text { and drank for } \\
\text { health tonic, } \\
\text { nervous } \\
\text { system. }\end{array}$ \\
\hline 54 & Som Phor & Streblus asper LOUR & & $\begin{array}{l}\text { Hua \&Tzen- } \\
\text { Yuh } 2017\end{array}$ & both & tree & $f$ & $\begin{array}{l}\text { The fresh fruit } \\
\text { are soaked with } \\
\text { warm water and } \\
\text { drank to } \\
\text { treating cough } \\
\text { or common } \\
\text { cold. }\end{array}$ \\
\hline 55 & Deau Pong & Ficus hispida L.f. & & $\begin{array}{l}\text { Lamxay et } \\
\text { al. } 2011\end{array}$ & both & tree & $\mathrm{t}$ & $\begin{array}{l}\text { Stem is boiled } \\
\text { and mixture } \\
\text { drank for bring } \\
\text { down fever. }\end{array}$ \\
\hline
\end{tabular}

Remake: vg* villagers, hl* healers; t, stem; l, leaves; r, roots; wh, whole plant; f, fruit; b, bark; In, liana. 


\begin{tabular}{|c|c|c|c|c|c|c|c|c|}
\hline No & Dialect names & Scientific name & Family name & References & $\mathrm{vg} / \mathrm{h} \mid * *$ & $\begin{array}{l}\text { Plant } \\
\text { form }\end{array}$ & $\begin{array}{l}\text { Part } \\
\text { used }\end{array}$ & $\begin{array}{l}\text { Method of } \\
\text { prepare }\end{array}$ \\
\hline 56 & Hang Kuang & $\begin{array}{l}\text { Gomphia serrata (Gaertn) } \\
\text { Kanis }\end{array}$ & Ochanaceae & $\begin{array}{l}\text { MoNRE- } \\
\text { IUCN, } 2016\end{array}$ & both & shrub & $\mathrm{t}$ & $\begin{array}{l}\text { The stem is } \\
\text { boiled and } \\
\text { mixture drank } \\
\text { for stomach } \\
\text { problems. }\end{array}$ \\
\hline 57 & Mai Mark Feung & Averhoa yarlambora L. & Oxalidaceae & $\begin{array}{l}\text { Southavong } \\
\text { et al. } 2014\end{array}$ & $\mathrm{vl}$ & tree & $\mathrm{r}$ & $\begin{array}{l}\text { Roots are boiled } \\
\text { and mixture } \\
\text { drank to } \\
\text { treating kidney } \\
\text { problems. }\end{array}$ \\
\hline 58 & Tong Teeb & Pandanus amaryllifilius & Pandanaceae & $\begin{array}{l}\text { Southavong } \\
\text { et al. } 2014\end{array}$ & $\mathrm{vl}$ & shrub & I & $\begin{array}{l}\text { Used } 3-5 \\
\text { leaves are } \\
\text { boiled and } \\
\text { drank to } \\
\text { treating nerve } \\
\text { pain/nervous } \\
\text { system. }\end{array}$ \\
\hline 59 & Trang Teep & $\begin{array}{l}\text { Glochidion brunneum } \\
\text { Hook. f. }\end{array}$ & Phyllanthaceae & $\begin{array}{l}\text { Southavong } \\
\text { et al. } 2014\end{array}$ & $\mathrm{hl}$ & Shrub & wh & $\begin{array}{l}\text { The whole plant } \\
\text { is boiled and } \\
\text { mixture drank } \\
\text { for stomach } \\
\text { problems. }\end{array}$ \\
\hline 60 & KharmPom & Phyllanthusemblica L. & & $\begin{array}{l}\text { Hua \&Tzen- } \\
\text { Yuh } 2017\end{array}$ & $\mathrm{vl}$ & tree & $r, l, f$ & $\begin{array}{l}\text { The stem and } \\
\text { leaves are } \\
\text { boiled and } \\
\text { drank or fresh } \\
\text { fruit eta directly } \\
\text { for stomach } \\
\text { problems. }\end{array}$ \\
\hline 61 & Deuy Hin & Coix aquatic & Poaceae & $\begin{array}{l}\text { Onvilay et } \\
\text { al. } 2016\end{array}$ & $\mathrm{hl}$ & shrub & $f$ & $\begin{array}{l}\text { Fruits are boiled } \\
\text { and mixture } \\
\text { drank to } \\
\text { treating kidney } \\
\text { problems. }\end{array}$ \\
\hline 62 & Mai KhoneTha & Harrisonia perforate & Rataceae & $\begin{array}{l}\text { Southavong } \\
\text { et al. } 2013\end{array}$ & both & shrub & r & $\begin{array}{l}\text { Roots are boiled } \\
\text { and mixture } \\
\text { drank for } \\
\text { stomach } \\
\text { problems. }\end{array}$ \\
\hline 63 & Seuakhong & $\begin{array}{l}\text { Ziziphus funiculosa } \\
\text { Ham. }\end{array}$ & Rhamnaceae & $\begin{array}{l}\text { Southavong } \\
\text { et al. } 2014\end{array}$ & both & liana & $\ln$ & $\begin{array}{l}\text { The liana cut is } \\
\text { a small piece } \\
\text { and soaked in } \\
\text { alcohol/boiled } \\
\text { drank for } \\
\text { infertility, } \\
\text { dysmenorhoea } \\
\text { or syncope }\end{array}$ \\
\hline 64 & Mai Pork & $\begin{array}{l}\text { Parinarium annamense } \\
\text { HANCE }\end{array}$ & Rocaceae & $\begin{array}{l}\text { Newman et } \\
\text { al. } 2007\end{array}$ & both & tree & $r$ & $\begin{array}{l}\text { Roots are boiled } \\
\text { and mixture } \\
\text { drank for } \\
\text { stomach } \\
\text { problems. }\end{array}$ \\
\hline 65 & Kok Khao & Haldina cordifolia Roxb. & Rubiaceae & $\begin{array}{l}\text { Onvilay et } \\
\text { al } 2016\end{array}$ & both & tree & $\mathrm{t}$ & $\begin{array}{l}\text { The stem cut is } \\
\text { a small piece } \\
\text { and boiled } \\
\text { drank for } \\
\text { stomach } \\
\text { problems }\end{array}$ \\
\hline 66 & Ya Ngoo Noy & Hedyotisdiffusa Willd. & & $\begin{array}{l}\text { Southavong } \\
\text { et al. } 2014\end{array}$ & both & gasses & wh & $\begin{array}{l}\text { Whole plant is } \\
\text { boiled drank for } \\
\text { health tonic. }\end{array}$ \\
\hline
\end{tabular}

Remake: vg* villagers, hl* healers; $t$, stem; l, leaves; $r$, roots; wh, whole plant; f, fruit; b, bark; In, liana. 


\begin{tabular}{|c|c|c|c|c|c|c|c|c|}
\hline No & Dialect names & Scientific name & Family name & References & $\mathrm{vg}^{\star} /\left.\mathrm{h}\right|^{\star \star *}$ & $\begin{array}{l}\text { Plant } \\
\text { form }\end{array}$ & $\begin{array}{l}\text { Part } \\
\text { used }\end{array}$ & $\begin{array}{l}\text { Method of } \\
\text { prepare }\end{array}$ \\
\hline 67 & Khat Koa & Randia simensis & & $\begin{array}{l}\text { Southavong } \\
\text { et al. } 2014\end{array}$ & both & tree & I & $\begin{array}{l}\text { Juice from the } \\
\text { crushed leaves } \\
\text { is used as a } \\
\text { dressing on } \\
\text { wounds. }\end{array}$ \\
\hline 68 & Som Kop & $\begin{array}{l}\text { Hymenodictyonexcelsum } \\
\text { WALL }\end{array}$ & & $\begin{array}{l}\text { Southavong } \\
\text { et al. } 2014\end{array}$ & both & tree & $r$ & $\begin{array}{l}\text { The roots are } \\
\text { soaked with } \\
\text { cold water and } \\
\text { drank to } \\
\text { treating fever. }\end{array}$ \\
\hline 69 & Som Sheun & $\begin{array}{l}\text { Glycosmis parvifolia } \\
\text { (simds) little }\end{array}$ & Rutacae & $\begin{array}{l}\text { Onvilay et } \\
\text { al. } 2016\end{array}$ & both & liana & $\ln$ & $\begin{array}{l}\text { The liana is } \\
\text { boiled and } \\
\text { mixture, drank } \\
\text { for treating } \\
\text { fever. }\end{array}$ \\
\hline 70 & Kok Ken & $\begin{array}{l}\text { Flacourtia rukam } \\
\text { Zoll.\&Mor. }\end{array}$ & Salicaceae & $\begin{array}{l}\text { Southavong } \\
\text { et al. } 2013\end{array}$ & both & tree & $r$ & $\begin{array}{l}\text { Roots are boiled } \\
\text { and mixture } \\
\text { drank to } \\
\text { treating } \\
\text { stomach } \\
\text { problems or } \\
\text { diarrhea. }\end{array}$ \\
\hline 71 & Nom Ngoua & $\begin{array}{l}\text { Scleropyrum pentandrum } \\
\text { (Dennst.) Mabb. }\end{array}$ & Santalaceae & $\begin{array}{l}\text { Onvilay et } \\
\text { al. } 2016\end{array}$ & both & tree & $r$ & $\begin{array}{l}\text { Roots are } \\
\text { soaked with } \\
\text { cold water } \\
\text { drank for the } \\
\text { postpartum } \\
\text { tonic. }\end{array}$ \\
\hline 72 & Wran Sa Noy & Scopariadulcis L. & Scrophulariaceae & $\begin{array}{l}\text { Southavong } \\
\text { et al. } 2013\end{array}$ & both & liana & $\ln$ & $\begin{array}{l}\text { The liana of } \\
\text { climber cut is a } \\
\text { small piece } \\
\text { mixture and } \\
\text { boiled drank for } \\
\text { alleviating easy } \\
\text { labour or weary }\end{array}$ \\
\hline 73 & Koun Tha & Harrisonia perforate & Simaroubaceae & $\begin{array}{l}\text { Southavong } \\
\text { et al. } 2014\end{array}$ & hl & tree & $\mathrm{t}$ & $\begin{array}{l}\text { Stem are boiled } \\
\text { and mixture } \\
\text { drank to } \\
\text { alleviating } \\
\text { jaundice. }\end{array}$ \\
\hline 74 & Bii Khon & Brucea sumatrana Roxb. & Simaroubaceae & $\begin{array}{l}\text { Southavong } \\
\text { et al. } 2014\end{array}$ & hl & shrub & $\mathrm{f}$ & $\begin{array}{l}\text { Dry fruit are } \\
\text { boiled and } \\
\text { drank treating } \\
\text { cholecystitis. }\end{array}$ \\
\hline 75 & ¿Ya Huoa & Smilax glabra Roxb & Smilacaceae & $\begin{array}{l}\text { Southavong } \\
\text { et al. 2013, } \\
2014\end{array}$ & both & liana & $r$ & $\begin{array}{l}\text { Roots are } \\
\text { boiled/soaked } \\
\text { with alcohol } \\
\text { drank to } \\
\text { improved } \\
\text { sexual } \\
\text { performance }\end{array}$ \\
\hline 76 & Kok Khai Doun & $\begin{array}{l}\text { Turpiniapomifera (Roxb.) } \\
\text { DC. }\end{array}$ & Staphyleaceae & $\begin{array}{l}\text { Southavong } \\
\text { et al. } 2013\end{array}$ & $\mathrm{hl}$ & tree & $\mathrm{I}, \mathrm{f}$ & $\begin{array}{l}\text { Juice leaves } \\
\text { crushed and } \\
\text { rubbed on the } \\
\text { body for skin } \\
\text { disease, and } \\
\text { fruit is crushed } \\
\text { as a powder } \\
\text { mixed with } \\
\text { warm water and } \\
\text { drank for heart } \\
\text { disease. }\end{array}$ \\
\hline
\end{tabular}

Remake: vg* villagers, hl* healers; $t$, stem; l, leaves; $r$, roots; wh, whole plant; f, fruit; b, bark; In, liana. 


\begin{tabular}{|c|c|c|c|c|c|c|c|c|}
\hline No & Dialect names & Scientific name & Family name & References & $\mathrm{vg} /\left.\mathrm{hl}\right|^{* *}$ & $\begin{array}{l}\text { Plant } \\
\text { form }\end{array}$ & $\begin{array}{l}\text { Part } \\
\text { used }\end{array}$ & $\begin{array}{l}\text { Method of } \\
\text { prepare }\end{array}$ \\
\hline 77 & Hum Aow & $\begin{array}{l}\text { pterospermum } \\
\text { semisagittatum }\end{array}$ & Sterculiaceae & $\begin{array}{l}\text { Newman et } \\
\text { al. } 2007\end{array}$ & both & tree & $\mathrm{t}$ & $\begin{array}{l}\text { The stem is } \\
\text { boiled and } \\
\text { drank for chest } \\
\text { pain. }\end{array}$ \\
\hline 78 & Pao Thong & Leptostachya sp & - & $\begin{array}{l}\text { Onvilay et } \\
\text { al. } 2016\end{array}$ & hl & tree & $\mathrm{t}$ & $\begin{array}{l}\text { The stem is } \\
\text { boiled and } \\
\text { mixed with cold } \\
\text { water and taken } \\
\text { like bath for } \\
\text { dysmenorrhea } \\
\text { or postpartum } \\
\text { tonic. }\end{array}$ \\
\hline 79 & Khii Mou Kheua & Castanopsis annamonsis & - & $\begin{array}{l}\text { Southavong } \\
\text { et al. } 2014\end{array}$ & $\mathrm{hl}$ & shrub & $\mathrm{t}$ & $\begin{array}{l}\text { The stem is } \\
\text { boiled and } \\
\text { drank for } \\
\text { treating } \\
\text { jaundice. }\end{array}$ \\
\hline
\end{tabular}

This research found that, the medicinal plant most usually used for the treatment of stomach problem (55.88\%), followed by kidney infection (23.53\%), fever (20.59\%), Dysmenorrheal/Postpartum tonic (17.65), skin disease and nervous system (14.71\%) each, health tonic and sexual performance (11.76\%) each, Liver disease and Jaundice (8.82\%), a weary and dressing wounds (5.88\%), and while the following complains, namely pain, cough and bruises, purgative, tonic blood, malaria, dizzy, emaciation, heart disease and sore throat (2.94\%) each (Fig. 5).

The main objective of this research was to keep the medicinal Plant knowledge in document form that used for treating different sickness since from ancient time and transfer orally from generation to the next generation. And the recording and maintaining of that knowledge in written form is also very useful for pharmaceutical purpose to developing advance medicine to treating these illnesses.

\subsection{Traditional Medicine Formulae}

Use for nervous system

\begin{tabular}{|lllll|}
\hline Species name & & & Part use & Dosage \\
Local name & Scientific name & Family & & \\
\hline SomLom & Aganonerionbpolymorphum & Apocynaceae & Liana & $10 \mathrm{~g}$ \\
\cline { 1 - 2 } Nga None Nai & Not identified & Fabaceae & Roots & $3 \mathrm{~g}$ \\
\hline NgaKhuoak & Desmodiumtriquetrum & Poaceae & Roots & $7 \mathrm{~g}$ \\
\hline Method of preparation: Take dried raw material and decoct in 1.5 liters of water. Drank as needed.
\end{tabular}

Use for kidney inflammation

\begin{tabular}{|lllll|}
\hline Species name & & \multicolumn{2}{l}{ Part use } & Dosage \\
\hline Local name & Scientific name & Family & \\
\hline Huoa-Eung & Costusspeiosus(Koecnig) & Costus & Stem & $2 \mathrm{~g}$ \\
\cline { 1 - 2 } NaoNarm & ArtabotrysspinosusCraib & Annonceae & Stem & $2 \mathrm{~g}$ \\
\hline Pao Thong & Leptostachyasp & Fabaceae & Roots & $2 \mathrm{~g}$ \\
\hline YaKhumPao & CarexbaccansNees & Cyperaceae & Roots & $1 \mathrm{~g}$ \\
\hline DeuayHin & Coix aquatic & Poaceae & Fruit & $0.05 \mathrm{~g}$ \\
\hline Sa Ly & Zea mays & Poaceae & Hair & $1 \mathrm{~g}$ \\
\hline SomLom & Aganonerionpolymorphum & Apocynaceae & Roots & $1 \mathrm{~g}$ \\
\hline Method of preparation: Take dried raw material and decoct in 2 liters of water, and drank as need. &
\end{tabular}

Use as postpartum tonic 


\begin{tabular}{|c|c|c|c|c|}
\hline \multicolumn{3}{|l|}{ Species name } & \multirow[t]{2}{*}{ Part use } & \multirow[t]{2}{*}{ Dosage } \\
\hline Local name & Scientific name & Family & & \\
\hline KokKhao & HaldinacordifoliaRoxb. & Rubiaceae & Roots & 1 root \\
\hline Nom Ngoua & Scleropyrumpentandrum(Dennst.)Mabb. & Santalaceae & Stem/Roots & 2 root \\
\hline ¿YaHuoa & Smilax glabraRoxb & Smilaceae & Roots & 1 root \\
\hline Sa Ly & Zea mays & Poaceae & Hair & $1 \mathrm{~g}$ \\
\hline HomSarmMeuang & Moghanialatifolia & Fabaceae & Root & 1 root \\
\hline Sa Math & Euodialepla & Rutaceae & Root & 1 root \\
\hline
\end{tabular}

\section{Conclusion}

It was found that the total 79 plant species that were used in traditional medicine in Hinboun district, Khammuoane province, Lao PDR. Of these a few species had high use values, suggesting that they may produce bioactive compounds with strong physiological effects. In this study, 79 medicinal plant species were documented as use in the treatment of different common sickness including stomach problem (gastrointestinal, flatulence, diarrhea, detox), kidney infections, fever, dysmenorrheal postpartum tonic, nervous system, skin disease, health tonic, lever infections, heart disease, dressing wounds, and others. The majority of these traditional medicinal plants are trees, shrubs, liana, and gasses that are mainly sourced from wild. Among the plant parts used, roots and stems are the most frequency used. The medicinal plants are prepared using dried plant parts while some other species are using in fresh form. Boiling, soaking with cold water or alcohol, crushing, and burning are main steps of preparation of medicine.

The current study also highlighted that realization and cultural beliefs have a significant influence on the unique source of healthcare, whereas religion was found to have no association with health-seeking behavior. Beyond, the immediate researchers believe that the therapeutic use of the identified and documented plants will provide basic data for further researches focus on pharmacological studies and the conservation of the most important medicinal plants in the study area.

\section{Declarations}

\section{- Ethics approval and consent to participate}

This paper our research teams from Faculty of Forestry Science National University of Laos. Plan worked was follow the role of Faculty and Province; by submitted the letter form Faculty to Province of Agriculture and Forestry Office, then province office will contract to District and from district to villages step by step. (supporting document on attract files or if need more detail contract to author by email)

\section{- Consent for publication}

Not applicable

\section{- Availability of data and materials}

Please contact author for data requests

\section{- Funding}

The program was financially supported by the Forestry Research Foundation for the Public Service from The State Forestry Administration of China," Research on Plant Diversity in Qinling Mountains"(2015,04320)

\section{- Competing interests}

The authors declare that they have no competing interests.

\section{- Authors' contributions}

Kang Yongxiang participated in the design of the study. Zhang Lily and Awais participated in the sequence alignment and drafted the manuscript. Souksamone and Channy conceived of the study and coordination and helped to data correction. Nishantha and Li Hua carried out the checking and ensure scientific name. All authors read and approved the final manuscript. 
We would like very grateful to teachers of Department of Forest Community and Rural Development at Faculty of Forestry Science, National University of Laos for providing us field materials, and their students to helping us in collection data. We would also like to express our gratitude to the Province of Forestry Office. Thankfully to villagers and healers for their invaluable contribution to the study, and also thanks to all the staff in department of forestry at Hinboun district for graciously granting us permission to conduct our study.

\section{References}

Abbas, Z., Khan, S.M., Abbasi, A.M., Pieroni, A., Ullah, Z., Iqbal, M., Ahmad, Z., 2016. Ethnobotany of the Balti community, Tormik valley, Karakorum range, Baltistan, Pakistan. J. Ethnobiol. Ethnomed.12 (1), 38.

Abbasi, A.M., Khan, M., Ahmad, M., Zafar, M., Jahan, S., Sultana, S., 2010. Ethno pharmacological application of medicinal plants to cure skin diseases and in folk cosmetics among the tribal communities of North-West Frontier Province, Pakistan. J. Ethnopharmacol. $128,322-335$.

Agbodeka, K., Gbekley, H.E., Karou, S.D., Anani, K., Agbonon, A., Tchacondo, T., Batawila, K., Simpore, J., Gbeassor, M., 2016.Ethnobotanical study of medicinal plants used for the treatment of malaria in the Plateau Region, Togo.Pharmacognosy Research 8, S12-S18.

Alemayehu, G., Asfaw, Z., Kelbessa, E., 2015. Ethnobotanical study of medicinal plants used by local communities of Minjar-Shenkora district, North Shewa zone of Amhara region, Ethiopia. J. Med. Plants Stud. 6, 01-11.

and remedies in ethnomedicine and ethnopharmacology. J. Ethnopharmacol. 174,

Asowata-Ayodele, A.M., Afolayan, A.J., Otunola, G.A., 2016. Ethnobotanical survey of culinary herbs and spices used in the traditional medicinal system of Nkonkobe Municipality, Eastern Cape, South Africa. South Afr. J. Bot. 104, 69-75.

Asowata-Ayodele, A.M., Afolayan, A.J., Otunola, G.A., 2016. Ethnobotanical survey of culinary herbs and spices used in the traditional medicinal system of Nkonkobe

Baruah, A., Bordoloi, M., DekaBaruah, H.P., 2016. Aloe vera: a multipurpose industrial

Birhanu, Z., Endale, A., Shewamene, Z., 2015. An ethnomedicinal investigation of plants used by traditional healers of Gondar town, NorthWestern Ethiopia. J. Med. Plants Stud. 3, 36-43.

Birru, E.M., Asrie, A.B., Adinew, G.M., Tsegaw, A., 2016. Antidiarrheal activity of crude methanolic root extract of IdigoferaspicataForssk. (Fabaceae). BMC Complement.Altern.Med.16 (1), 272.

Bolson, M., Hefler, S.R., DallıOglio Chaves, E.I., Gasparotto Junior, A., Cardozo Junior,E.L., 2015. Ethno-medicinal study of plants used for treatment of human ailments, with residents of the surrounding region of forest fragments of Paraná, Brazil. J.Ethnopharmacol. 161, 1-10.

Chekole, G., 2017. Ethnobotanical study of medicinal plants used against human ailments in Gubalafto District, Northern Ethiopia. J. Ethnobiol. Ethnomed. 13,55

Chekole, G., Asfaw,Z., Kelbessa,E., 2015. Ethnobotanical study of medicinal plantsin the environs of Tara-gedam and Amba remnant forest of LiboKemken district, northwest Ethiopia. J. Ethnobiol. Ethnomed 11,4.

crop. Ind. Crops Prod. 94, 951-963.

De Boer, H., \&Lamxay, V. (2009). Plants used during pregnancy, childbirth and postpartum healthcare in Lao PDR: A comparative study of the Brou, Saek and Kry ethnic groups. Journal of Ethnobiology and Ethnomedicine, 5(1), 25.

Delang, C. O. (2007). The role of medicinal plants in the provision of health care in Lao PDR. Journal of Medicinal Plants Research, 1(3), 050059.

Dhama, K., Karthik, K., Khandia, R., Munjal, A., Tiwari, R., Rana, R., ...\&Farag, M. R. (2018). Medicinal and therapeutic potential of herbs and plant metabolites/extracts countering viral pathogens-current knowledge and future prospects. Current drug metabolism, 19(3), 236-263.

Elking, B.G; Southavong, B; Sydara, K; Souliya,O; Vanthanouvong, M; Nettavong, K; Thammachack, B; Dennis, H. Pak, Riley, M.C; Franzblau, S.G; Soejarto, D.D. 2009. Biological Evaluation of plants of Laos used in the Treatment of Tuberculosis in Lao Traditional Medicine Pharmaceutical (Formerly international journal of Pharmacognosy), Volume 47, Number 1, pp. 26-33 
ethnobiology in Southeast Asia. J. Ethnobiol. Ethnomed. 11.

Fabricant, D.S., Farnsworth, N.R., 2001. The value of plants used in traditional medicine for drug discovery. Environ. Health Perspect. 109 (Suppl 1), 69-75.

Fortini, P., Di Marzio, P., Guarrera, P.M., lorizzi, M., 2016. Ethnobotanical study on the medicinal plants in the Mainarde Mountains (centralsouthern Apennine, Italy). J. Ethnopharmacol. 184, 208-218.

GadisaDemie, MeseleNegash, TesfayeAwas., 2018. Ethnobotanical study of medicinal plants used by indigenous people in and around Dirre Sheikh Hussein heritage site of South-eastern Ethniopia. Journal of Ethnopharmacology; 220:87-03

Girmay, T., Testhome, Z., 2017. Assessment of traditional Medicinal plants used to treat human and livestock ailments and their threatening factors in Gulomekeda District, Northern Ethiopia. IJETST 04, 5061-5070.

Hidayati, S., Franco, F.M., Bussmann, R.W., 2015. Ready for phase 5 - current status of

Hua Z, Tzen-Yuh C 2017. Floristic characteristics and affinities in Lao PDR, with a reference to the biogeography of the Indochina peninsula[J]. PLOS ONE, 12(6): e0179966-.

Iwu, M.M., 2002. Chapter 25 - Ethnobotanical approach to pharmaceutical drug discovery: strengths and limitations. In: Maurice, M.I., Jacqueline, C.W. (Eds.), Advances in Phytomedicine. Elsevier, pp. 309-320.

Jahandideh, M., Hajimehdipoor, H., Mortazavi, Sa, dehpour, A., Hassanzadeh, G., 2016.

Jai-aue, A., Makchuchit, S., Juckmeta, T., Itharat, A., 2014. Anti-allergic, anti-inflammatory and antioxidant activities of the different extracts of Thai traditionalremedy called prabchompoothaweep for allergic rhinitis treatment. J. Med. Assoc. Thai 97 (Suppl. 8), S140-S148.

Kichu, M., Malewska, T., Akter, K., Imchen, I., Harrington, D., Kohen, J., Vemulpad, S.R.,Jamie, J.F., 2015. An ethnobotanical study of medicinal plants of Chungtia village, Nagaland, India. J. Ethnopharmacol. 166, 5-17.

Lamxay,V., de Boer, H.J., Bjork, L., 2011. Traditions and plant use during pregnancy, childbirth and postpartum recovery by the Kry ethnic group in Lao PDR.J.Ethnobiol. Ethnomed.7 (1), 14.

Lu, Y., Hernandez, P., Abegunde, D., \&Edejer, T. (2011). The world medicines situation 2011. Medicine expenditures. World Health Organization, Geneva.

MahwasaneMiddleton L., Boaduo N., 2013. An ethnobotanical survey of indigenous knowledge on medicinal plants used by the traditional healers of the Lwamondo area, Limpopo province, South Africa. Journal of Botany 88, 69-75.

McFarland, B., Bigelow, D., Zani, B., Newsom, J., Kaplan, M., 2002.Complementary and alternative medicine use in Canada and the United States. Am. J. Public Health 92 (10), 1616-1618.

Menale, B., De Castro, O., Cascone, C., Muoio, R., 2016. Ethnobotanical investigation on medicinal plants in the Vesuvio National Park (Campania, Southern Italy). J. Ethnopharmacol. 192, 320-349.

MetheePhumthum, KamonnateSrithi, Angkhanalnta, AuempornJunsongduang, KornkanokTangjitman, WittayaPongamornkul, ChusieTrisonthi, HenrikBalslev., 2018. Ethnobotanical plant diversity in Thailand. Journal of Ethnopharmacology 214; 90-98.

Molassiotis, A., Fernadez-Ortega, P., Pud, D., Ozden, G., Scott, J.A., Panteli, V., Margulies, A., Browall, M., Magri, M., Selvekerova, S., 2005. Use of complementary and alternative medicine in cancer patients: a European survey. Ann. Oncol. 16 (4), 655-663.

Mukungu, N., Abuga, K., Okalebo, F., Ingwela, R., Mwangi, J., 2016. Medicinal plantsused for management of malaria among the Luhya community of Kakamega East sub-County, Kenya. J. Ethnopharmacol. 194, 98-107.

Municipality, Eastern Cape, South Africa. South Afr. J. Bot. 104, 69-75.

Newman M, Ketphanh S, Svengsuksa B, et al., 2007. A checklist of the vascular plants of Lao PDR. [M].

Onvilay, S., Kheithisack, P., Sang Woo, L., 2016. Medicinal and Food Plants in Dongsouth-Donglong Forest Area in Phoukoutdostrict, Xiangkhouang Province, Lao PDR. Institute of Traditional Medicine, Volume I. Ministry of Health, Vientiane Capital, Lao PDR. 
Ouelbani, R., Bensari, S., Mouas, T.N., Khelifi, D., 2016. Ethnobotanical investigations on plants used in folk medicine in the regions of Constantine and Mila (North-East of Algeria). J. Ethnopharmacol. 194, 196-218.

Park, J. H., Kang, H. S., Bang, M., Cheng, H. C., Jin, H. Y., Ahn, T. H., \&Phongoudome, C. (2018). Floristic inventory of vascular plant in Nam Ha national biodiversity conservation area, Lao people's democratic republic. Journal of Asia-Pacific Biodiversity, 11(2), 300-304.

Pieroni, A., Sõukand, R., Quave, C.L., Hajdari, A., Mustafa, B., 2017. Traditional food usesof wild plants among the Gorani of South Kosovo. Appetite 108, 83-92.

Pooma, R., Suddee, S., 2014. Tem Smitinand's Thai Plant Names, revised. The Office of

Ragupathy, S., Steven, N. G., Maruthakkutti, M., Velusamy, B., \&Ul-Huda, M. M. (2008). Consensus of the'Malasars' traditional aboriginal knowledge of medicinal plants in the Velliangiri holy hills, India. Journal of Ethnobiology and Ethnomedicine, 4(1), 8.

Ramet, A., Benyei, P., Parada, M., Aceituno-Mata, L., García-del-Amo, D., \& Reyes-García, V. (2018). Grandparents' proximity and children's traditional medicinal plant knowledge: Insights from two schools in intermediate-rural Spain. Journal of ethnobiology, 38(2), $187-205$.

Singh, D., Baghel, U.S., Gautam, A., Baghel, D.S., Yadav, D., Malik, J., Yadav, R., 2016.The genus Anogeissus: a review on ethnopharmacology, phytochemistry and pharmacology.J. Ethnopharmacol. 194, 30-56.

Southavong B, Sydara K, Souliya O, et al. 2013. Medicinal plants and herbs in the Lao People's Democratic Republic Volume I. Vientiane, Lao PDR: Institute of Traditional Medicine of Ministry of Public Health. p. 399.

Southavong B, Sydara K, Souliya O, et al. 2014. Medicinal plants and herbs in the Lao People's Democratic Republic Volume II. Vientiane, Lao PDR: Institute of Traditional Medicine of Ministry of Public Health. p. 309.

Srithi, K., Balslev, H., Wangpakapattanawong, P., Srisanga, P., \&Trisonthi, C. (2009). Medicinal plant knowledge and its erosion among the Mien (Yao) in northern Thailand. Journal of ethnopharmacology, 123(2), 335-342.

Staub, P.O., Geck, M.S., Weckerle, C.S., Casu, L., Leonti, M., 2015. Classifying diseases

the Forest Herbarium, Department of National Parks, Wildlife and Plant Conservation, Bangkok.

Yemele, M.D., Telefo, P.B., Lienou, L.L., Tagne, S.R., Fodouop, C.S.P., Goka, C.S., Lemfack, M.C., Moundipa, F.P., 2015. Ethnobotanical survey of medicinal plants used for pregnant women $\square$ s health conditions in Menoua division-West Cameroon. J. Ethnopharmacol. 160, $14-31$.

\section{Figures}

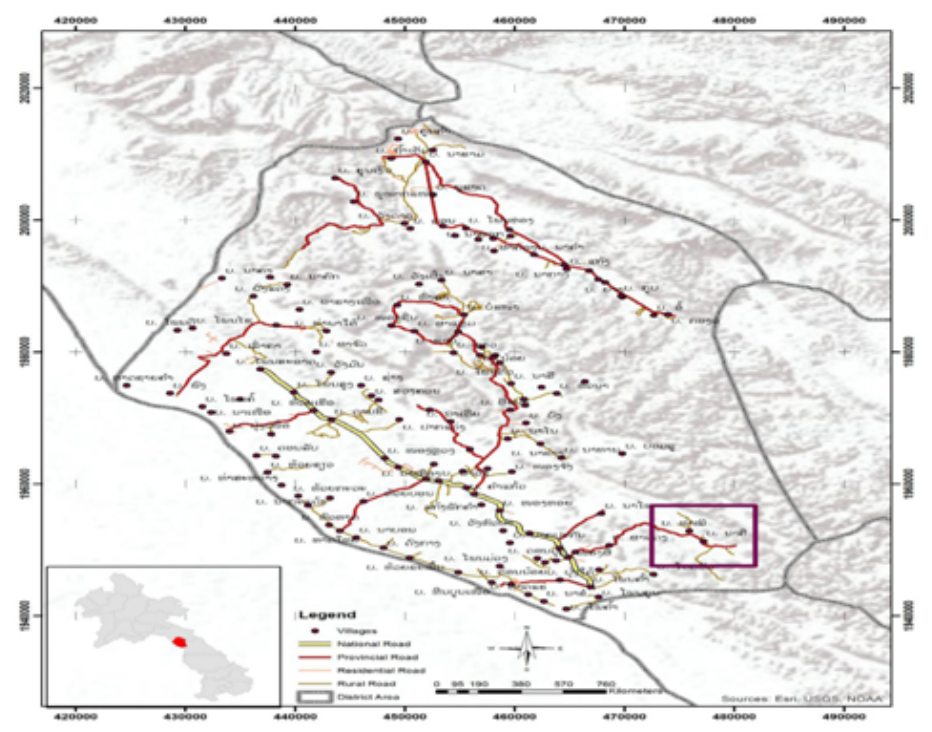

\section{Figure 1}

Map showing Hinboun district, Nakeu village study area 


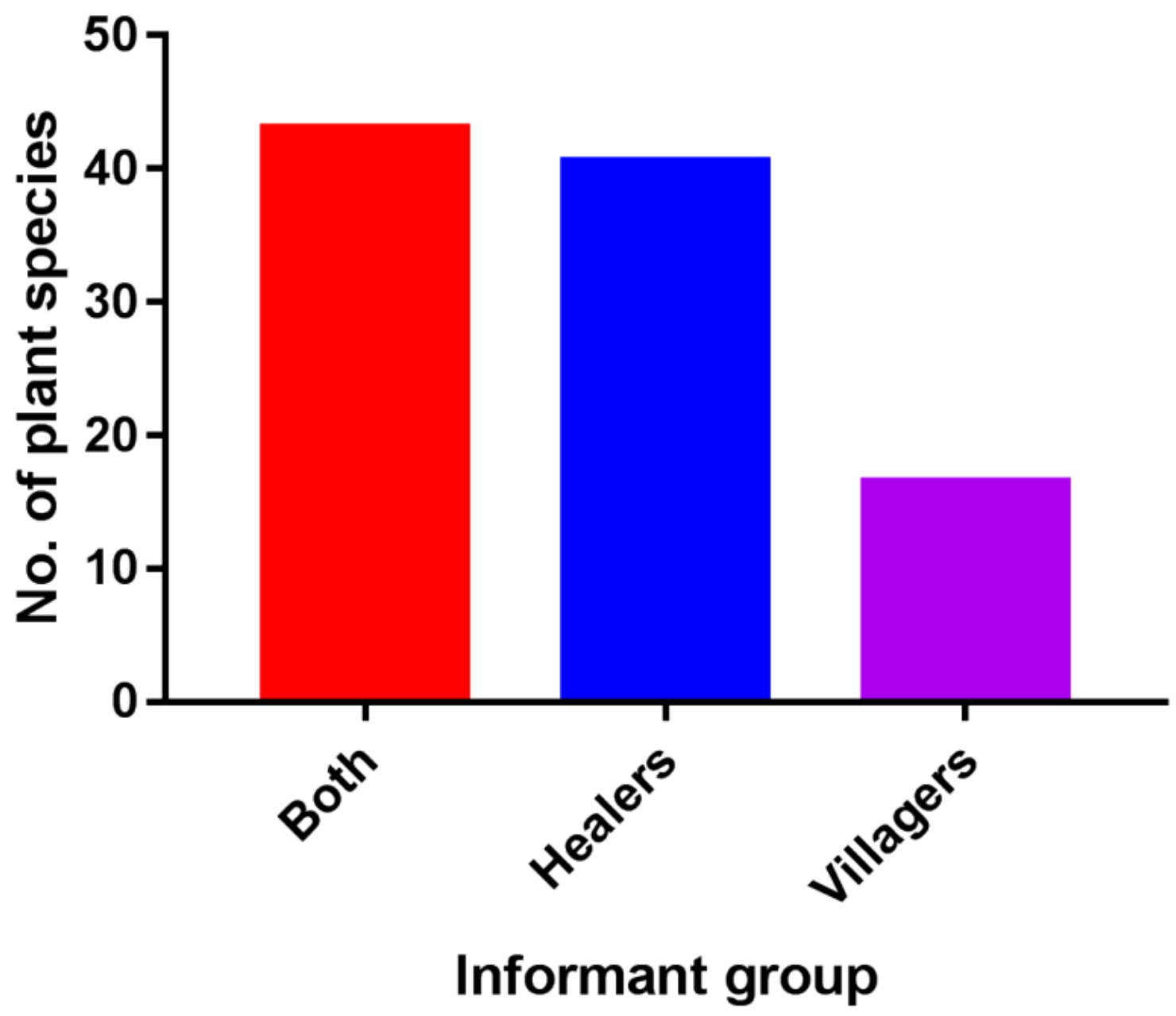

Figure 2

Number of plant species that knew by healers and villagers in Hinboun district, Khammouane province. 


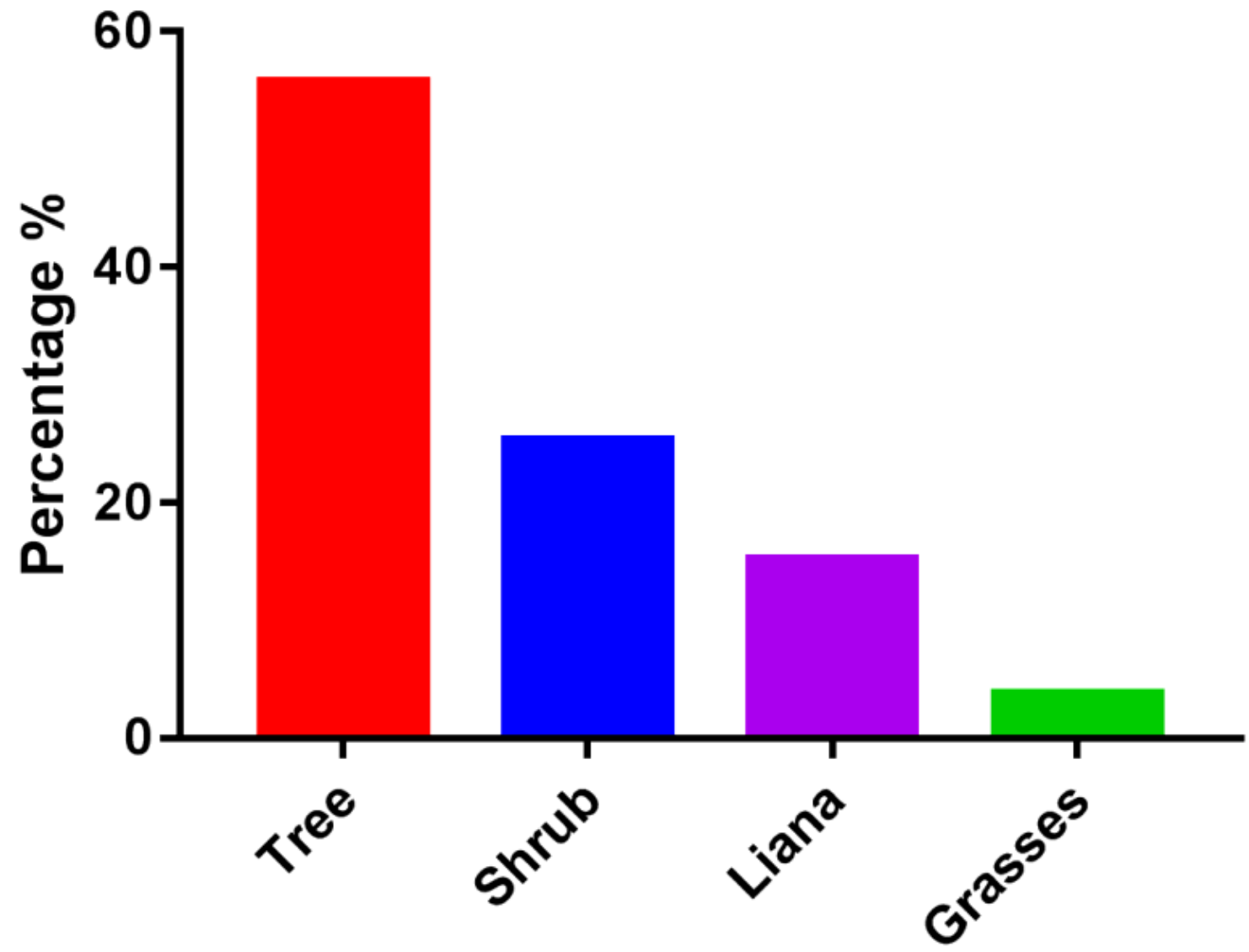

Plant form

\section{Figure 3}

Plant form representation of medicinal plant in Hinboun district, Khammouane province.

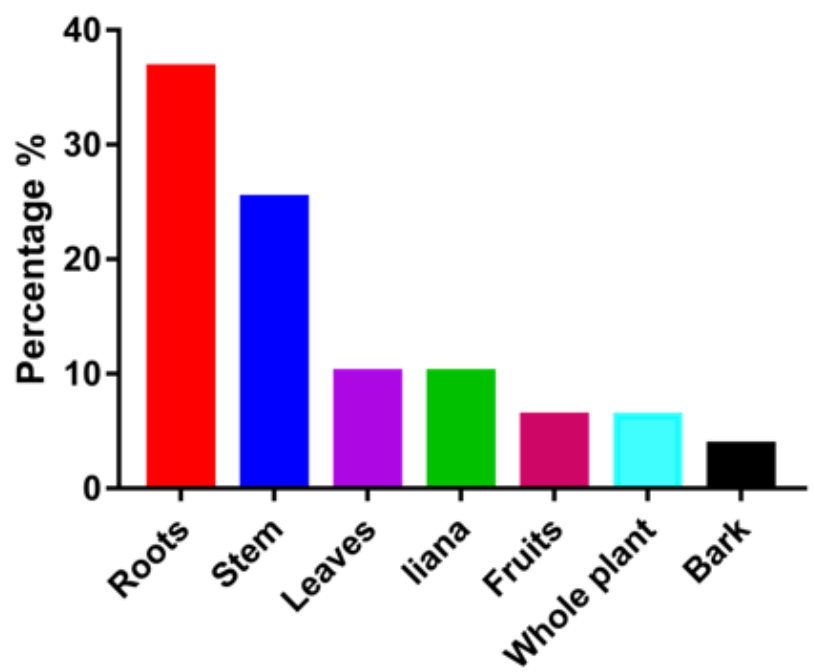

Plant parts used

Figure 4 
Percentage use of plant part use in Hinboun district, Khammouane province.

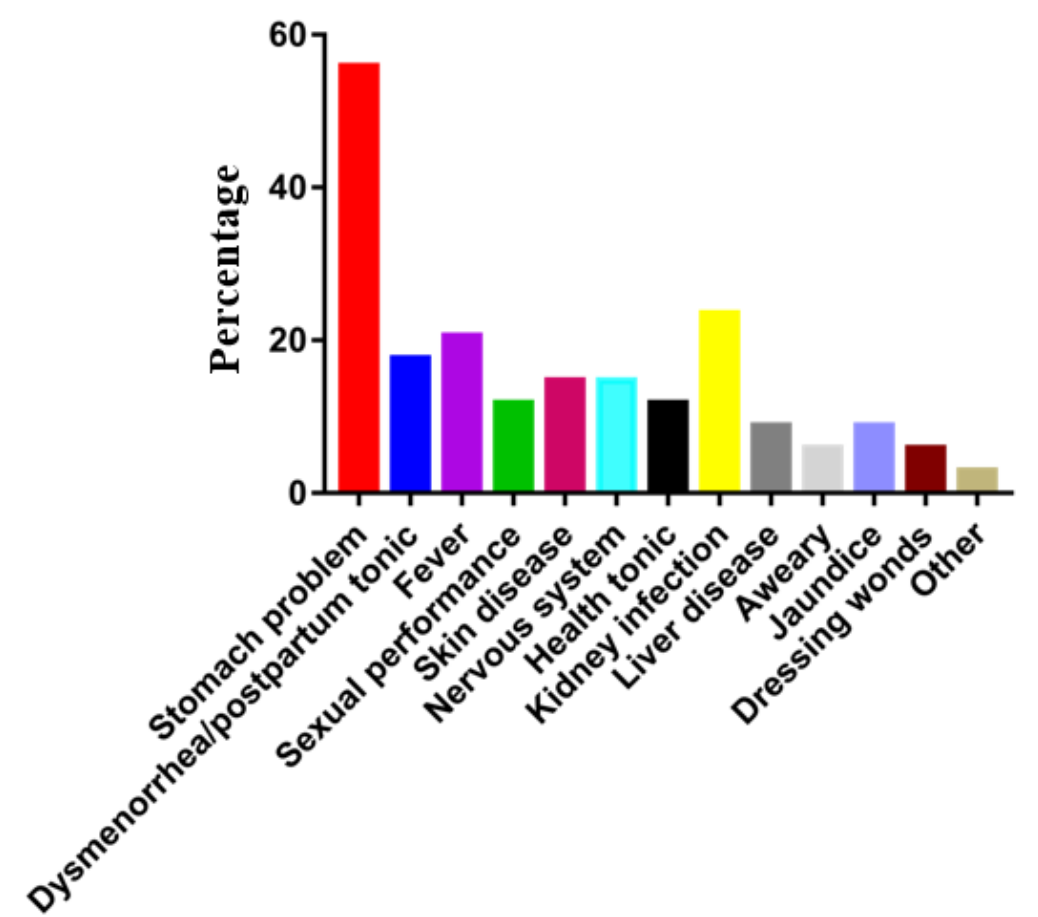

Disease condition

\section{Figure 5}

The frequency by percentage of the type's medicine conditions treating by the medicinal plants used in Hinboun district, Khammouane province.

\section{Supplementary Files}

This is a list of supplementary files associated with this preprint. Click to download.

- Supportingdocument1.jpg

- Supportingdocument1.jpg

- Suporting4.PNG

- Suporting4.PNG

- Suporting3.PNG

- Suporting3.PNG

- Suporting2.PNG

- Suporting2.PNG

- Suporting1.PNG

- Suporting1.PNG 